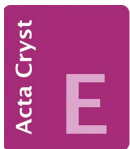

CRYSTALLOGRAPHIC COMMUNICATIONS

ISSN 2056-9890
Received 3 November 2020

Accepted 2 December 2020

Edited by S. Parkin, University of Kentucky, USA

Keywords: crystal structure; dihydro-

quinoxaline; hydrogen bond.

CCDC reference: 2047850

Supporting information: this article has supporting information at journals.iucr.org/e
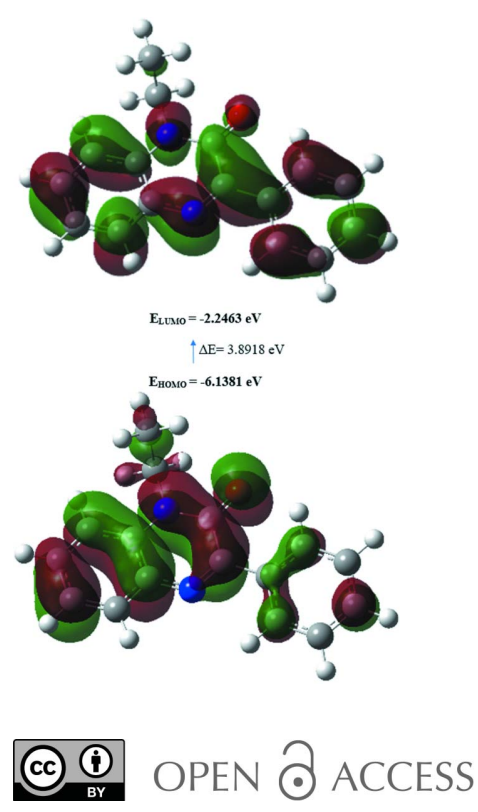

\section{Crystal structure, Hirshfeld surface analysis and DFT study of 1-ethyl-3-phenyl-1,2-dihydro- quinoxalin-2-one}

\author{
Gamal Al Ati, ${ }^{\text {Karim Chkirate, }}{ }^{\mathrm{a}}$ Ashraf Mashrai, ${ }^{\mathrm{b} *}$ Joel T. Mague, ${ }^{\mathrm{c}}$ Youssef Ramli, ${ }^{\mathrm{d}}$ \\ Redouane Achour ${ }^{\mathrm{a}}$ and El Mokhtar Essassi ${ }^{\mathrm{a}}$
}

\begin{abstract}
aLaboratory of Heterocyclic Organic Chemistry URAC 21, Pharmacochemistry Competence Center, Av. Ibn Battouta, BP 1014, Faculty of Sciences, Mohammed V University, Rabat, Morocco, ${ }^{\mathbf{b}}$ Department of Pharmacy, University of Science and Technology, Ibb Branch, Ibb, Yemen, '⿳D Department of Chemistry, Tulane University, New Orleans, LA 70118, USA, and ${ }^{\mathbf{d}}$ Laboratory of Medicinal Chemistry, Drug Sciences Research Center, Faculty of Medicine and Pharmacy, Mohammed V University in Rabat, Morocco. *Correspondence e-mail: ashraf.yemen7@gmail.com
\end{abstract}

In the title molecule, $\mathrm{C}_{16} \mathrm{H}_{14} \mathrm{~N}_{2} \mathrm{O}$, the dihydroquinoxaline moiety is not planar as there is a dihedral angle of $4.51(5)^{\circ}$ between the constituent rings. In the crystal, $\mathrm{C}-\mathrm{H}$... O hydrogen bonds form helical chains about the crystallographic $2_{1}$ screw axis in the $b$-axis direction. Hirshfeld surface analysis indicates that the most important contributions to the crystal packing are from $\mathrm{H} \cdots \mathrm{H}(51.7 \%)$, $\mathrm{H} \cdots \mathrm{C} / \mathrm{C} \cdot \mathrm{H}(26 \%)$ and $\mathrm{H} \cdots \mathrm{O} / \mathrm{O} \cdots \mathrm{H}(8.5 \%)$ interactions. The optimized structure calculated using density functional theory (DFT) at the B3LYP/6$311 \mathrm{G}(\mathrm{d}, \mathrm{p})$ level is compared with the experimentally determined structure in the solid state. The calculated HOMO-LUMO energy gap is $3.8918 \mathrm{eV}$.

\section{Chemical context}

Nitrogen-based structures have attracted attention in recent years because of their interesting properties in structural and inorganic chemistry (Chkirate et al., 2019; 2020a,b). The family of nitrogenous drugs, particularly those containing the quinoxaline moiety, is important in medicinal chemistry because of their wide range of pharmacological activities, which include anticancer, anti-inflammatory, antibacterial, antituberculosis, anti-glycation, anti-analgesic and antifungal properties, and for their antioxidant potential. In particular, quinoxalin-2-one derivatives are active anti-tumor agents with tyrosine kinase receptor inhibition properties (Galal et al., 2014). They can also selectively antagonize the glycoprotein in cancer cells (Sun et al., 2009). Quinoxalin-2-one derivatives are also potential antagonist ligands for imaging the A2A adenosine receptor by positron emission tomography (PET) (Holschbach et al., 2005). Given the wide range of therapeutic applications for such compounds, we have already reported a route for the preparation of quinoxalin-2-one derivatives using $N$-alkylation reactions carried out with di-halogenated carbon chains (Benzeid et al., 2011); a similar approach yielded the title compound, $\mathrm{C}_{16} \mathrm{H}_{14} \mathrm{~N}_{2} \mathrm{O}$, (I). In addition to the synthesis, we also report the molecular and crystal structure along with a Hirshfeld surface analysis and a density functional theory (DFT) computational study carried out at the B3LYP/6-311 G(d,p) level. 


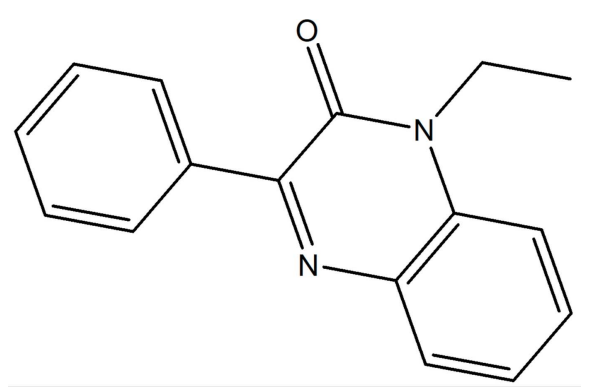

\section{Structural commentary}

The molecular structure of (I) is depicted in Fig. 1. The dihydroquinoxaline moiety is not planar, as indicated by the dihedral angle of $4.51(5)^{\circ}$ between the constituent rings. Alternatively, the maximum deviations from the mean plane (r.m.s. deviation $=0.060 \AA$ ) of the ten-membered, fused ring system are 0.096 (1) $\AA$ (C8) and -0.057 (1) $\AA$ (C7). The mean planes of the $\mathrm{C} 11-\mathrm{C} 16$ and $\mathrm{C} 1 / \mathrm{C} 6 / \mathrm{N} 1 / \mathrm{C} 7 / \mathrm{C} 8 / \mathrm{N} 2$ rings are inclined to one another by $30.87(4)^{\circ}$. The $\mathrm{C} 6-\mathrm{N} 1-\mathrm{C} 9-\mathrm{C} 10$ torsion angle is $-78.78(10)^{\circ}$, indicating the ethyl substituent is rotated well out of the plane of the dihydroquinoxaline moiety (Fig. 1).

\section{Supramolecular features}

In the crystal, helical chains about the crystallographic $2_{1}$ axes are formed by $\mathrm{C} 9-\mathrm{H} 9 B \cdots \mathrm{O} 1$ hydrogen bonds (Table 1 , Figs. 2 and 3). The chains pack via normal van der Waals contacts.

\section{Hirshfeld surface}

In order to visualize the intermolecular interactions in the crystal of the title compound, a Hirshfeld surface (HS) analysis (Hirshfeld, 1977) was carried out using Crystal Explorer 17.5 (Turner et al., 2017). A view of the three-

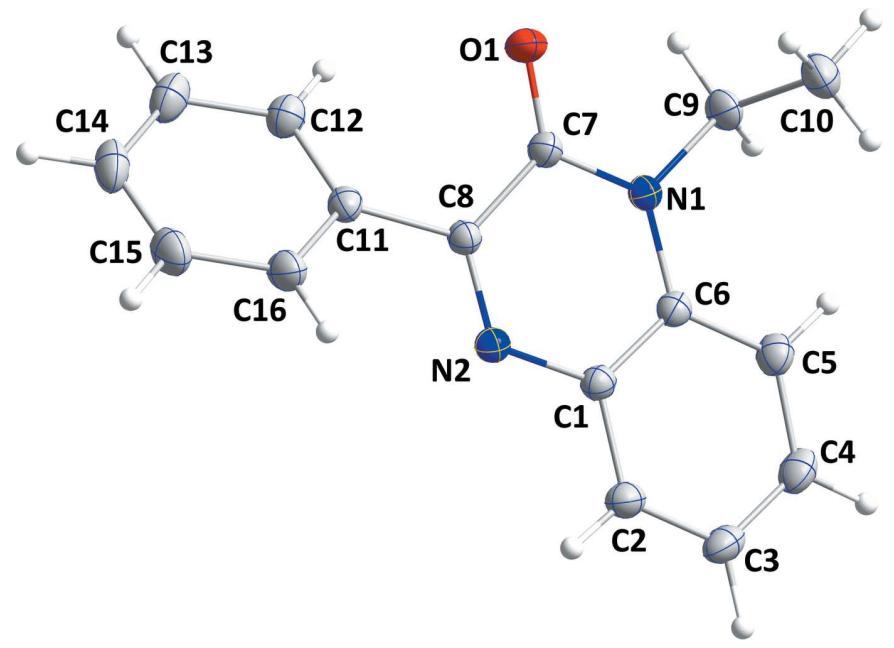

Figure 1

The title molecule with the atom-labelling scheme and $50 \%$ probability ellipsoids.
Table 1

Hydrogen-bond geometry $\left(\AA{ }^{\circ}\right)$.

\begin{tabular}{lllll}
\hline$D-\mathrm{H} \cdots A$ & $D-\mathrm{H}$ & $\mathrm{H} \cdots A$ & $D \cdots A$ & $D-\mathrm{H} \cdots A$ \\
\hline $\mathrm{C} 9-\mathrm{H} 9 B \cdots \mathrm{O} 1^{\mathrm{i}}$ & $0.975(13)$ & $2.396(13)$ & $3.3340(11)$ & $161.3(10)$ \\
\hline
\end{tabular}

Symmetry code: (i) $-x+\frac{1}{2}, y-\frac{1}{2},-z+\frac{1}{2}$.

dimensional Hirshfeld surface of (I), plotted over $d_{\text {norm }}$ is shown in Fig. 4. The overall two-dimensional fingerprint plot (McKinnon et al., 2007) is shown in Fig. 5a, while those delineated into $\mathrm{H} \cdots \mathrm{H}, \mathrm{H} \cdots \mathrm{C} / \mathrm{C} \cdots \mathrm{H}, \mathrm{H} \cdots \mathrm{N} / \mathrm{N} \cdots \mathrm{H}, \mathrm{H} \cdots \mathrm{O} /$ $\mathrm{O} \cdots \mathrm{H}, \mathrm{C} \cdots \mathrm{C}, \mathrm{C} \cdots \mathrm{N} / \mathrm{N} \cdots \mathrm{C}$ and $\mathrm{C} \cdots \mathrm{O} / \mathrm{O} \cdots \mathrm{C}$ contacts are illustrated in Fig. $5 b-h$, respectively, together with their relative contributions to the Hirshfeld surface. The most important interactions are $\mathrm{H} \cdots \mathrm{H}$, contributing $51.7 \%$ to the overall crystal packing, which is reflected in Fig. $5 b$ as widely scattered points of high density due to the large hydrogen content of the

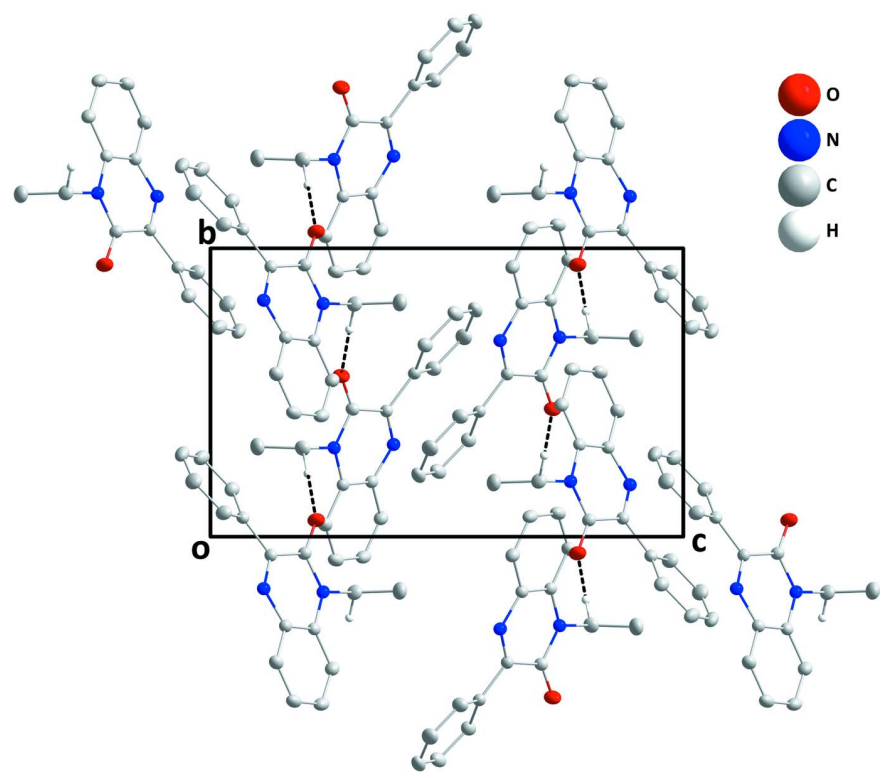

Figure 2

Packing view along the $a$-axis direction with $\mathrm{C}-\mathrm{H} \cdots \mathrm{O}$ hydrogen bonds shown as dashed lines.

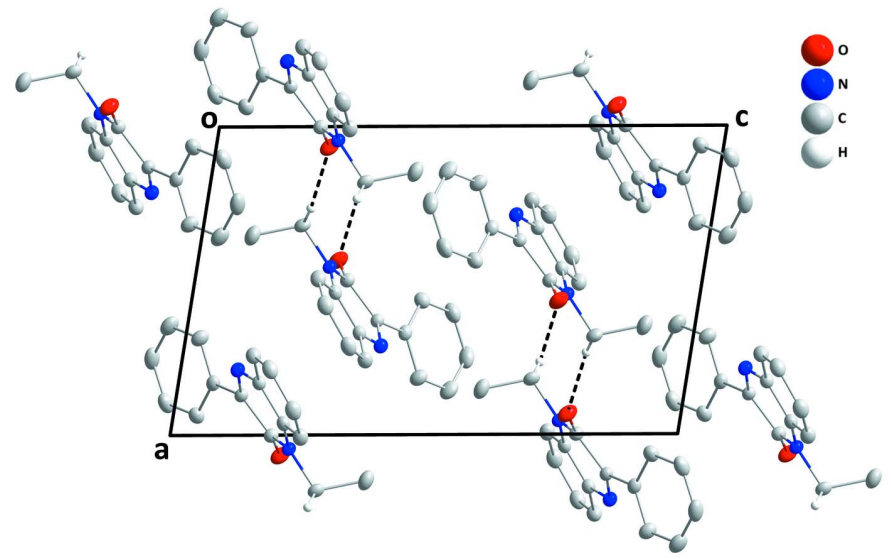

Figure 3

Packing view along the $b$-axis direction with $\mathrm{C}-\mathrm{H} \cdots \mathrm{O}$ hydrogen bonds shown as dashed lines. 


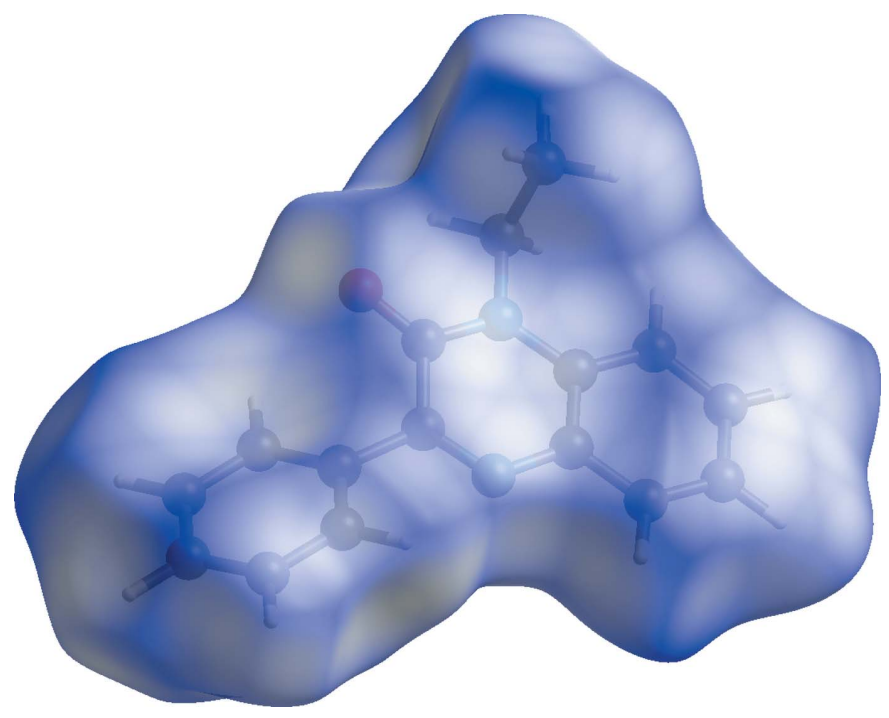

Figure 4

View of the three-dimensional Hirshfeld surface of the title compound, plotted over $d_{\text {norm }}$.

molecule, with the tip at $d_{\mathrm{e}}=d_{\mathrm{i}}=1.07 \AA$. For $\mathrm{C}-\mathrm{H}$ interactions, the pair of characteristic wings in the fingerprint plot delineated into $\mathrm{H} \cdots \mathrm{C} / \mathrm{C} \cdots \mathrm{H}$ contacts $(26 \%$ contribution to the HS), Fig. $5 c$, have tips at $d_{\mathrm{e}}+d_{\mathrm{i}}=2.79 \AA$. The pair of scattered points of spikes in the fingerprint plot delineated into $\mathrm{H} \cdots \mathrm{O} / \mathrm{O} \cdots \mathrm{H}$, Fig. $5 e(8.5 \%)$, have the tips at $d_{\mathrm{e}}+d_{\mathrm{i}}=$ $2.26 \AA$. The C . . C contacts, Fig. $5 f(6.1 \%)$, have the tips at

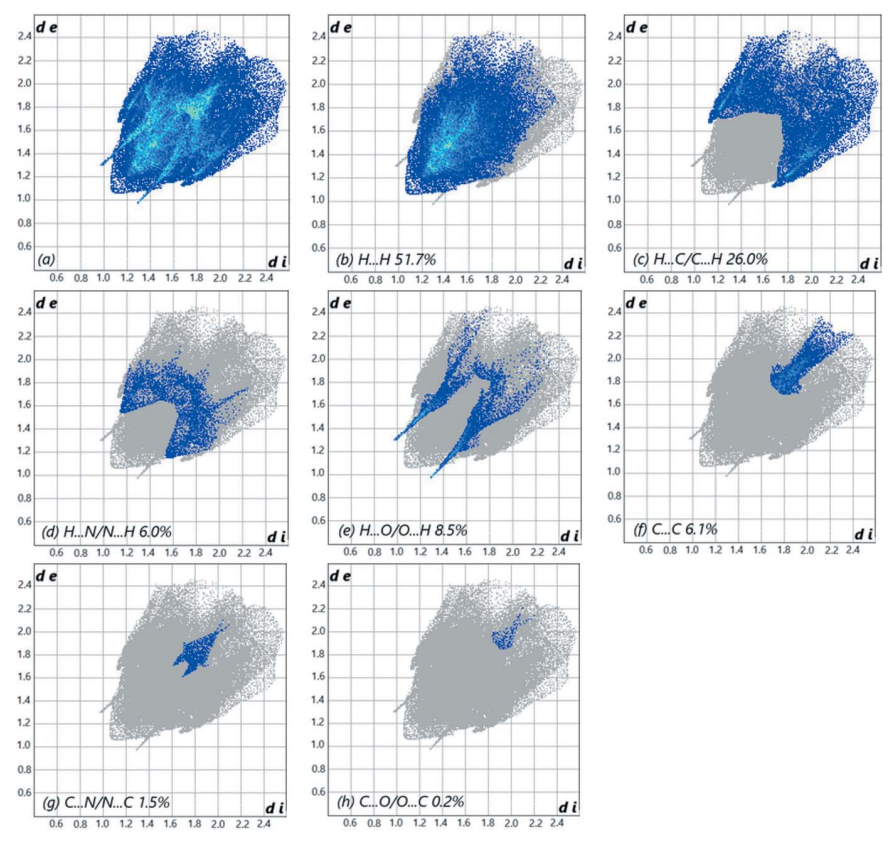

Figure 5

The full two-dimensional fingerprint plots for the title compound, showing $(a)$ all interactions, and delineated into $(b) \mathrm{H} \cdots \mathrm{H},(c) \mathrm{H} \cdots \mathrm{C} /$ $\mathrm{C} \cdots \mathrm{H},(d) \mathrm{H} \cdots \mathrm{N} / \mathrm{N} \cdots \mathrm{H},(e) \mathrm{H} \cdots \mathrm{O} / \mathrm{O} \cdots \mathrm{H},(f) \mathrm{C} \cdots \mathrm{C},(g) \mathrm{C} \cdots \mathrm{N} / \mathrm{N} \cdots \mathrm{C}$ and $(h) \mathrm{C} \cdots \mathrm{O} / \mathrm{O} \cdots \mathrm{C}$ interactions. The $d_{\mathrm{i}}$ and $d_{\mathrm{e}}$ values are the closest internal and external distances (in $\AA$ ) from points on the Hirshfeld surface.
Table 2

Comparison of selected (X-ray and DFT) bond lengths and angles $\left(\AA{ }^{\circ}\right)$.

\begin{tabular}{lll}
\hline & X-ray & B3LYP/6-311G(d,p) \\
\hline $\mathrm{C} 1-\mathrm{C} 6$ & $1.4071(12)$ & 1.4149 \\
$\mathrm{~N} 2-\mathrm{C} 1$ & $1.3846(11)$ & 1.3724 \\
$\mathrm{~N} 2-\mathrm{C} 8$ & $1.2983(11)$ & 1.299 \\
$\mathrm{C} 8-\mathrm{C} 11$ & $1.4864(11)$ & 1.486 \\
$\mathrm{C} 7-\mathrm{C} 8$ & $1.4872(11)$ & 1.4949 \\
$\mathrm{O} 1-\mathrm{C} 7$ & $1.2299(10)$ & 1.2235 \\
$\mathrm{~N} 1-\mathrm{C} 7$ & $1.3791(10)$ & 1.3974 \\
$\mathrm{~N} 1-\mathrm{C} 9$ & $1.4732(11)$ & 1.4745 \\
$\mathrm{C} 9-\mathrm{C} 10$ & $1.5156(14)$ & 1.5289 \\
$\mathrm{~N} 1-\mathrm{C} 6$ & $1.3936(10)$ & 1.3893 \\
$\mathrm{C} 6-\mathrm{N} 1-\mathrm{C} 9$ & $120.63(7)$ & 121.2759 \\
$\mathrm{C} 7-\mathrm{N} 1-\mathrm{C} 6$ & $122.16(7)$ & 122.6246 \\
$\mathrm{C} 7-\mathrm{N} 1-\mathrm{C} 9$ & $117.14(7)$ & 116.0858 \\
$\mathrm{C} 8-\mathrm{N} 2-\mathrm{C} 1$ & $119.29(7)$ & 120.9715 \\
$\mathrm{O} 1-\mathrm{C} 7-\mathrm{N} 1$ & $121.54(8)$ & 120.1959 \\
$\mathrm{O} 1-\mathrm{C} 7-\mathrm{C} 8$ & $123.36(7)$ & 124.593 \\
$\mathrm{~N} 1-\mathrm{C} 9-\mathrm{C} 10$ & $111.67(7)$ & 112.8427 \\
$\mathrm{~N} 1-\mathrm{C} 6-\mathrm{C} 5$ & $122.78(8)$ & 123.4659 \\
$\mathrm{~N} 2-\mathrm{C} 8-\mathrm{C} 11$ & $117.24(7)$ & \\
\hline
\end{tabular}

Table 3

Calculated energies.

\begin{tabular}{lr}
\hline Molecular property & Compound (I) \\
\hline Total energy $T E(\mathrm{eV})$ & -21853.0851 \\
$E_{\mathrm{HOMO}}(\mathrm{eV})$ & -6.1381 \\
$E_{\mathrm{LUMO}}(\mathrm{eV})$ & -2.2463 \\
Gap, $\Delta E(\mathrm{eV})$ & 3.8918 \\
Dipole moment, $\mu($ Debye $)$ & 3.0212 \\
Ionization potential, $I(\mathrm{eV})$ & 6.1381 \\
Electron affinity, $A$ & 2.2463 \\
Electronegativity, $\chi$ & 4.1922 \\
Hardness, $\eta$ & 1.9459 \\
Electrophilicity, index $\omega$ & 4.5158 \\
Softness, $\sigma$ & 0.5139 \\
Fraction of electrons transferred, $\Delta N$ & 0.7215 \\
\hline
\end{tabular}

$d_{\mathrm{e}}+d_{\mathrm{i}}=3.45 \AA$ A. The $\mathrm{H} \cdots \mathrm{N} / \mathrm{N} \cdots \mathrm{H}$ contacts, Fig. $5 d$, contribute $6 \%$ to the HS and appear as a pair of scattered points of spikes with the tips at $d_{\mathrm{e}}+d_{\mathrm{i}}=2.67 \AA$. The C $\cdots \mathrm{N} / \mathrm{N} \cdots \mathrm{C}$ contacts, Fig. $5 g$, contribute $1.5 \%$ to the HS, appearing as pair of scattered points of spikes with the tips at $d_{\mathrm{e}}+d_{\mathrm{i}}=3.30 \AA$. Finally, the C $\cdots \mathrm{O} / \mathrm{O} \cdots \mathrm{C}$ contacts, Fig. $5 h$, make only a $0.2 \%$ contribution to the HS and have a low-density distribution of points.

\section{DFT calculations}

The optimized structure of (I) in the gas phase was calculated by density functional theory (DFT) using a standard B3LYP functional and the 6-311 G(d,p) basis-set (Becke, 1993) as implemented in GAUSSIAN 09 (Frisch et al., 2009). The theoretical and experimental results related to bond lengths and angles are in good agreement (Table 2). Calculated numerical values for (I) including electronegativity $(\chi)$, hardness $(\eta)$, ionization potential $(I)$, dipole moment $(\mu)$, electron affinity $(A)$, electrophilicity $(\omega)$ and softness $(\sigma)$ are collated in Table 3. The electron transition from the HOMO to the LUMO energy level is shown in Fig. 6. The HOMO and LUMO are localized in the plane extending over the whole 

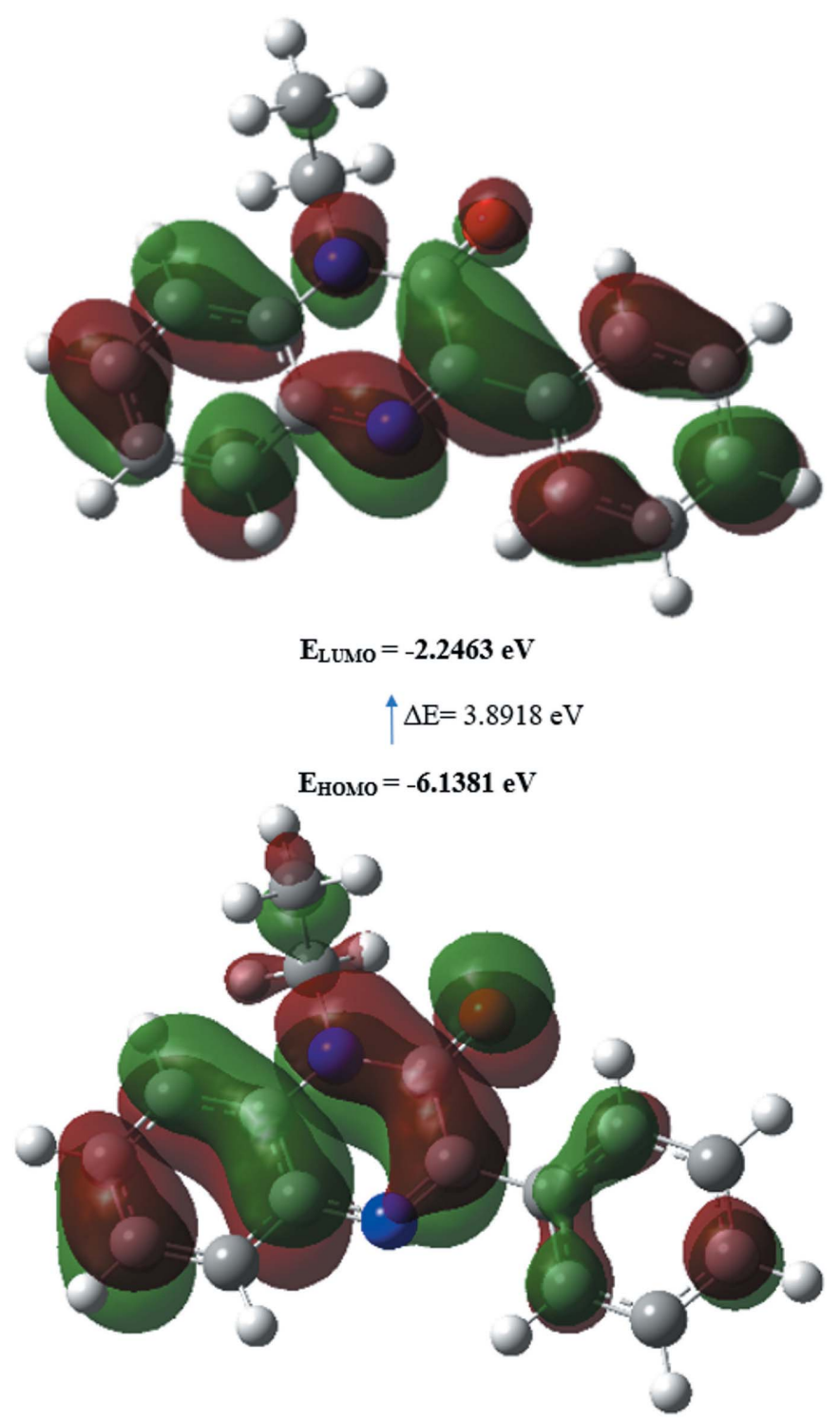

Figure 6

The energy band gap of (I).

1-ethyl-3-phenyl-1,2-dihydroquinoxalin-2-one system. The energy band gap $\left[\Delta E=E_{\mathrm{LUMO}}-E_{\mathrm{HOMO}}\right]$ of the molecule is $3.8918 \mathrm{eV}$, and the frontier molecular orbital energies, $E_{\mathrm{HOMO}}$ and $E_{\mathrm{LUMO}}$, are -6.1381 and $-2.2463 \mathrm{eV}$, respectively.

\section{Database survey}

A search of the Cambridge Structural Database (CSD version 5.40, updated March 2020; Groom et al., 2016) with the quinoxaline-2-one fragment yielded multiple matches. Of these, two had a phenyl at position 3 and are thus most comparable to (I). The first [(II), refcode NIBXEE; Abad et al., 2018a)] has (oxiran-2-yl) methyl on nitrogen 1, and the second [(III), IDOSUR; Daouda et al., 2013)] has a 3-ethyloxazolidin-2-one on nitrogen 1 (Fig. 7). Other structures having the quinoxaline-2-one moiety were observed by changing the substituents of positions 1 and 3 in the examples NAYTAJ (1-ethyl; Mamedov et al., 2005a), DUSHUV01

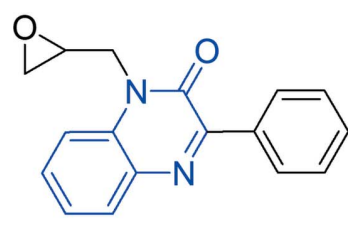

II<smiles>O=C1OCCN1CCn1c(=O)c(-c2ccccc2)nc2ccccc21</smiles>

III
Figure 7

Structures similar to (I): (II) (CSD refcode NIBXEE) and (III) (CSD refcode IDOSUR) obtained in the database search. The search fragment is indicated in blue.

(1-benzyl-3-methyl; Ramli et al., 2018), DUMRUB \{1-([1-(3azido-2-hydroxypropyl)-1 $H$-1,2,3-triazol-4-yl]methyl)-3-methyl; Abad et al., 2020\}, HIRZOA \{1- [(1-butyl-1H-1,2,3-triazol4-yl)methyl]-3-methyl; Abad et al., 2018b\} and SENYUG [3- (indolizin-2-yl)-1-ethyl; Mamedov et al., 2005b]. The dihedral angle between the dihydroquinoxaline ring system and the phenyl ring is $28.4(2)^{\circ}$ in NIBXEE and the $\mathrm{N}-\mathrm{C}-\mathrm{C}-\mathrm{O}$ torsion angle is $87.8(5)^{\circ}$; the mean plane through the fusedring system forms a dihedral angle of $30.72(5)^{\circ}$ with the attached phenyl ring. The molecular conformation is enforced by $\mathrm{C}-\mathrm{H} \cdots \mathrm{O}$ hydrogen bonds in IDOSUR. In (I), the dihydroquinoxaline moiety is not planar, as indicated by the dihedral angle of $4.51(5)^{\circ}$ between the constituent rings. The phenyl ring is tilted towards the pyrazine ring by $30.87(4)^{\circ}$, which is approximately the same as in IDOSUR but more tilted than in NIBXEE.

\section{Synthesis and crystallization}

To a solution of 3-phenylquinoxalin-2(1H)-one $(0.7 \mathrm{~g}$, $0.0032 \mathrm{~mol})$ in $N, N$-dimethylformamide $(20 \mathrm{ml})$ were added bromoethane $(0.48 \mathrm{ml})$, potassium carbonate $\mathrm{K}_{2} \mathrm{CO}_{3}(0.5 \mathrm{~g}$, $0.004 \mathrm{~mol}$ ) and a catalytic quantity of tetra- $n$-butylammonium bromide. The reaction mixture was stirred at room temperature for $24 \mathrm{~h}$. The solution was filtered and the solvent removed under reduced pressure. The residue thus obtained was separated by chromatography on a silica gel column using a hexane/ethyl acetate 9:1 mixture as eluent. The solid obtained was recrystallized from ethanol solution to afford colourless plates of the title compound (yield: $85 \%$ ).

\section{Refinement}

Crystal data, data collection and structure refinement details are summarized in Table 4. Hydrogen atoms were included as riding contributions in idealized positions $(\mathrm{C}-\mathrm{H}=0.95-$ $0.99 \AA$ ̊) with $U_{\text {iso }}(\mathrm{H})=1.2 U_{\text {eq }}(\mathrm{C})$ or $1.5 U_{\text {eq }}(\mathrm{C}$-methyl $)$.

\section{Funding information}

JTM thanks Tulane University for support of the Tulane Crystallography Laboratory. 
Table 4

Experimental details.

\begin{tabular}{|c|c|}
\hline \multicolumn{2}{|l|}{ Crystal data } \\
\hline Chemical formula & $\mathrm{C}_{16} \mathrm{H}_{14} \mathrm{~N}_{2} \mathrm{O}$ \\
\hline$M_{\mathrm{r}}$ & 250.29 \\
\hline Crystal system, space group & Monoclinic, $P 2_{1} / n$ \\
\hline Temperature $(\mathrm{K})$ & 150 \\
\hline$a, b, c(\AA)$ & 9.2572 (9), 9.0531 (9), 15.0557 (14) \\
\hline$\beta\left(^{\circ}\right)$ & $99.329(1)$ \\
\hline$V\left(\AA^{3}\right)$ & $1245.1(2)$ \\
\hline$Z$ & 4 \\
\hline Radiation type & Мо $K \alpha$ \\
\hline$\mu\left(\mathrm{mm}^{-1}\right)$ & 0.09 \\
\hline Crystal size $(\mathrm{mm})$ & $0.50 \times 0.47 \times 0.16$ \\
\hline \multicolumn{2}{|l|}{ Data collection } \\
\hline Diffractometer & Bruker SMART APEX CCD \\
\hline Absorption correction & $\begin{array}{l}\text { Multi-scan (SADABS; Krause et } \\
\quad \text { al., 2015) }\end{array}$ \\
\hline$T_{\min }, T_{\max }$ & $0.96,0.99$ \\
\hline $\begin{array}{l}\text { No. of measured, independent and } \\
\text { observed }[I>2 \sigma(I)] \text { reflections }\end{array}$ & $23365,3364,2925$ \\
\hline$R_{\text {int }}$ & 0.025 \\
\hline$(\sin \theta / \lambda)_{\max }\left(\AA^{-1}\right)$ & 0.688 \\
\hline \multicolumn{2}{|l|}{ Refinement } \\
\hline$R\left[F^{2}>2 \sigma\left(F^{2}\right)\right], w R\left(F^{2}\right), S$ & $0.042,0.130,1.09$ \\
\hline No. of reflections & 3364 \\
\hline No. of parameters & 228 \\
\hline H-atom treatment & All $\mathrm{H}$-atom parameters refined \\
\hline$\Delta \rho_{\max }, \Delta \rho_{\min }\left(\mathrm{e} \AA^{-3}\right)$ & $0.41,-0.21$ \\
\hline
\end{tabular}

Computer programs: APEX3 and SAINT (Bruker, 2016), SHELXT (Sheldrick, 2015a), SHELXL2018/1 (Sheldrick, 2015b), DIAMOND (Brandenburg \& Putz, 2012) and SHELXTL (Sheldrick, 2008).

\section{References}

Abad, N., El Bakri, Y., Sebhaoui, J., Ramli, Y., Essassi, E. M. \& Mague, J. T. (2018a). IUCrData, 3, x180610.

Abad, N., Hajji, M., Ramli, Y., Belkhiria, M., Moftah, H., Elmgirhi, S. A., Habib, M., Guerfel, T. T., Mague, J. T. \& Essassi, E. M. (2020). J. Phys. Org. Chem. 33, e4055.

Abad, N., Ramli, Y., Hökelek, T., Sebbar, N. K., Mague, J. T. \& Essassi, E. M. (2018b). Acta Cryst. E74, 1815-1820.

Becke, A. D. (1993). J. Chem. Phys. 98, 5648-5652.

Benzeid, H., Bouhfid, R., Massip, S., Leger, J. M. \& Essassi, E. M. (2011). Acta Cryst. E67, o2990.

Brandenburg, K. \& Putz, H. (2012). DIAMOND, Crystal Impact GbR, Bonn, Germany.

Bruker (2016). APEX3, SADABS and SAINT. Bruker AXS Inc., Madison, Wisconsin, USA.

Chkirate, K., Fettach, S., El Hafi, M., Karrouchi, K., Elotmani, B., Mague, J. T., Radi, S., Faouzi, M. E. A., Adarsh, N. N., Essassi, E. M. \& Garcia, Y. (2020a). J. Inorg. Biochem. 208, 111092.
Chkirate, K., Fettach, S., Karrouchi, K., Sebbar, N. K., Essassi, E. M., Mague, J. T., Radi, S., Faouzi, M. E. A., Adarsh, N. N. \& Garcia, Y. (2019). J. Inorg. Biochem. 191, 21-28.

Chkirate, K., Karrouchi, K., Dege, N., Sebbar, N. K., Ejjoummany, A., Radi, S., Adarsh, N. N., Talbaoui, A., Ferbinteanu, M., Essassi, E. M. \& Garcia, Y. (2020b). New J. Chem. 44, 2210-2221.

Daouda, B., Doumbia, M. L., Essassi, E. M., Saadi, M. \& El Ammari, L. (2013). Acta Cryst. E69, o662.

Frisch, M. J., Trucks, G. W., Schlegel, H. B., Scuseria, G. E., Robb, M. A., Cheeseman, J. R., Scalmani, G., Barone, V., Mennucci, B., Petersson, G. A., Nakatsuji, H., Caricato, M., Li, X., Hratchian, H. P., Izmaylov, A. F., Bloino, J., Zheng, G., Sonnenberg, J. L., Hada, M., Ehara, M., Toyota, K., Fukuda, R., Hasegawa, J., Ishida, M., Nakajima, T., Honda, Y., Kitao, O., Nakai, H., Vreven, T., Montgomery, J. A. Jr, Peralta, J. E., Ogliaro, F., Bearpark, M., Heyd, J. J., Brothers, E., Kudin, K. N., Staroverov, V. N., Kobayashi, R., Normand, J., Raghavachari, K., Rendell, A., Burant, J. C., Iyengar, S. S., Tomasi, J., Cossi, M., Rega, N., Millam, J. M., Klene, M., Knox, J. E., Cross, J. B., Bakken, V., Adamo, C., Jaramillo, J., Gomperts, R., Stratmann, R. E., Yazyev, O., Austin, A. J., Cammi, R., Pomelli, C., Ochterski, J. W., Martin, R. L., Morokuma, K., Zakrzewski, V. G., Voth, G. A., Salvador, P., Dannenberg, J. J., Dapprich, S., Daniels, A. D., Farkas, O., Foresman, J. B., Ortiz, J. V., Cioslowski, J. \& Fox, D. J. (2009). GAUSSIAN09. Rev. A.02. Gaussian Inc., Wallingford, CT, USA.

Galal, S. A., Khairat, S. H. M., Ragab, F. A. F., Abdelsamie, A. S., Ali, M. M., Soliman, S. M., Mortier, J., Wolber, G. \& El Diwani, H. I. (2014). Eur. J. Med. Chem. 86, 122-132.

Groom, C. R., Bruno, I. J., Lightfoot, M. P. \& Ward, S. C. (2016). Acta Cryst. B72, 171-179.

Hirshfeld, H. L. (1977). Theor. Chim. Acta, 44, 129-138.

Holschbach, M. H., Bier, D., Wutz, W., Sihver, W., Schüller, M. \& Olsson, R. A. (2005). Eur. J. Med. Chem. 40, 421-437.

Krause, L., Herbst-Irmer, R., Sheldrick, G. M. \& Stalke, D. (2015). J. Appl. Cryst. 48, 3-10.

Mamedov, V. A., Kalinin, A. A., Gubaidullin, A. T., Isaikina, O. G. \& Litvinov, I. A. (2005a). Zh. Org. Khim. 41, 609-616.

Mamedov, V. A., Kalinin, A. A., Yanilkin, V. V., Gubaidullin, A. T., Latypov, Sh. K., Balandina, A. A., Isaikina, O. G., Toropchina, A. V., Nastapova, N. V., Iglamova, N. A. \& Litvinov, I. A. (2005b). Izv. Akad. Nauk, Ser. Khim. 11, 2534-2542.

McKinnon, J. J., Jayatilaka, D. \& Spackman, M. A. (2007). Chem. Commun. pp. 3814.

Ramli, Y., El Bakri, Y., El Ghayati, L., Essassi, E. M. \& Mague, J. T. (2018). IUCrData, 3, x180390.

Sheldrick, G. M. (2008). Acta Cryst. A64, 112-122.

Sheldrick, G. M. (2015a). Acta Cryst. A71, 3-8.

Sheldrick, G. M. (2015b). Acta Cryst. C71, 3-8.

Sun, L.-R., Li, X., Cheng, Y.-N., Yuan, H.-Y., Chen, M.-H., Tang, W., Ward, S. G. \& Qu, X.-J. (2009). Biomed. Pharmacother. 63, 202-208.

Turner, M. J., McKinnon, J. J., Wolff, S. K., Grimwood, D. J., Spackman, P. R., Jayatilaka, D. \& Spackman, M. A. (2017). CrystalExplorer17. The University of Western Australia. 


\section{supporting information}

Acta Cryst. (2021). E77, 18-22［https://doi.org/10.1107/S2056989020015819]

\section{Crystal structure, Hirshfeld surface analysis and DFT study of 1-ethyl-3-} phenyl-1,2-dihydroquinoxalin-2-one

\section{Gamal Al Ati, Karim Chkirate, Ashraf Mashrai, Joel T. Mague, Youssef Ramli, Redouane Achour and El Mokhtar Essassi}

\section{Computing details}

Data collection: APEX3 (Bruker, 2016); cell refinement: SAINT (Bruker, 2016); data reduction: SAINT (Bruker, 2016); program(s) used to solve structure: SHELXT (Sheldrick, 2015a); program(s) used to refine structure: SHELXL2018/1 (Sheldrick, 2015b); molecular graphics: DIAMOND (Brandenburg \& Putz, 2012); software used to prepare material for publication: SHELXTL (Sheldrick, 2008).

1-Ethyl-3-phenyl-1,2-dihydroquinoxalin-2-one

\section{Crystal data}

$\mathrm{C}_{16} \mathrm{H}_{14} \mathrm{~N}_{2} \mathrm{O}$

$M_{r}=250.29$

Monoclinic, $P 2_{1} / n$

$a=9.2572(9) \AA$

$b=9.0531(9) \AA$

$c=15.0557(14) \AA$

$\beta=99.329(1)^{\circ}$

$V=1245.1(2) \AA^{3}$

$Z=4$

\section{Data collection}

Bruker SMART APEX CCD diffractometer

Radiation source: fine-focus sealed tube Graphite monochromator

Detector resolution: 8.3333 pixels $\mathrm{mm}^{-1}$ $\varphi$ and $\omega$ scans

Absorption correction: multi-scan

(SADABS; Krause et al., 2015)

$T_{\min }=0.96, T_{\max }=0.99$

\section{Refinement}

Refinement on $F^{2}$

Least-squares matrix: full

$R\left[F^{2}>2 \sigma\left(F^{2}\right)\right]=0.042$

$w R\left(F^{2}\right)=0.130$

$S=1.09$

3364 reflections

228 parameters

0 restraints
$F(000)=528$

$D_{\mathrm{x}}=1.335 \mathrm{Mg} \mathrm{m}^{-3}$

Mo $K \alpha$ radiation, $\lambda=0.71073 \AA$

Cell parameters from 9889 reflections

$\theta=2.4-29.2^{\circ}$

$\mu=0.09 \mathrm{~mm}^{-1}$

$T=150 \mathrm{~K}$

Plate, colourless

$0.50 \times 0.47 \times 0.16 \mathrm{~mm}$

23365 measured reflections

3364 independent reflections

2925 reflections with $I>2 \sigma(I)$

$R_{\text {int }}=0.025$

$\theta_{\text {max }}=29.3^{\circ}, \theta_{\min }=2.4^{\circ}$

$h=-12 \rightarrow 12$

$k=-12 \rightarrow 12$

$l=-20 \rightarrow 20$

Primary atom site location: difference Fourier map

Secondary atom site location: difference Fourier map

Hydrogen site location: difference Fourier map

All $\mathrm{H}$-atom parameters refined

$w=1 /\left[\sigma^{2}\left(F_{\mathrm{o}}^{2}\right)+(0.0911 P)^{2}+0.1012 P\right]$

where $P=\left(F_{\mathrm{o}}^{2}+2 F_{\mathrm{c}}^{2}\right) / 3$ 
$(\Delta / \sigma)_{\max }<0.001$

$\Delta \rho_{\max }=0.41$ e $\AA^{-3}$

$\Delta \rho_{\min }=-0.21 \mathrm{e} \AA^{-3}$

Special details

Experimental. The diffraction data were obtained from 3 sets of 400 frames, each of width $0.5^{\circ}$ in $\omega$, collected at $\varphi=$ $0.00,90.00$ and $180.00^{\circ}$ and 2 sets of 800 frames, each of width $0.45^{\circ}$ in $\varphi$, collected at $\omega=-30.00$ and $210.00^{\circ}$. The scan time was $10 \mathrm{sec} /$ frame.

Geometry. All esds (except the esd in the dihedral angle between two 1.s. planes) are estimated using the full covariance matrix. The cell esds are taken into account individually in the estimation of esds in distances, angles and torsion angles; correlations between esds in cell parameters are only used when they are defined by crystal symmetry. An approximate (isotropic) treatment of cell esds is used for estimating esds involving l.s. planes.

Refinement. Refinement of $\mathrm{F}^{2}$ against ALL reflections. The weighted R-factor $\mathrm{wR}$ and goodness of fit $\mathrm{S}$ are based on $\mathrm{F}^{2}$, conventional $\mathrm{R}$-factors $\mathrm{R}$ are based on $\mathrm{F}$, with $\mathrm{F}$ set to zero for negative $\mathrm{F}^{2}$. The threshold expression of $\mathrm{F}^{2}>2 \operatorname{sigma}\left(\mathrm{F}^{2}\right)$ is used only for calculating R-factors(gt) etc. and is not relevant to the choice of reflections for refinement. R-factors based on $\mathrm{F}^{2}$ are statistically about twice as large as those based on $\mathrm{F}$, and R- factors based on ALL data will be even larger.

Fractional atomic coordinates and isotropic or equivalent isotropic displacement parameters $\left(\AA^{2}\right)$

\begin{tabular}{lllll}
\hline & $x$ & $y$ & $z$ & $U_{\text {iso }} / U_{\text {eq }}$ \\
\hline O1 & $0.43347(7)$ & $0.55741(7)$ & $0.27640(5)$ & $0.02811(19)$ \\
N1 & $0.45495(8)$ & $0.30830(7)$ & $0.26163(5)$ & $0.01831(17)$ \\
N2 & $0.71192(8)$ & $0.31976(7)$ & $0.38726(5)$ & $0.01948(18)$ \\
C1 & $0.66994(9)$ & $0.19050(9)$ & $0.34103(6)$ & $0.01852(19)$ \\
C2 & $0.75776(10)$ & $0.06426(9)$ & $0.36083(6)$ & $0.0229(2)$ \\
H2 & $0.8512(14)$ & $0.0771(12)$ & $0.4013(9)$ & $0.030(3)^{*}$ \\
C3 & $0.71308(11)$ & $-0.07042(10)$ & $0.32364(6)$ & $0.0254(2)$ \\
H3 & $0.7731(13)$ & $-0.1609(13)$ & $0.3384(9)$ & $0.029(3)^{*}$ \\
C4 & $0.57963(11)$ & $-0.08094(10)$ & $0.26512(7)$ & $0.0262(2)$ \\
H4 & $0.5463(15)$ & $-0.1782(14)$ & $0.2407(10)$ & $0.040(4)^{*}$ \\
C5 & $0.49306(10)$ & $0.04224(10)$ & $0.24245(6)$ & $0.0237(2)$ \\
H5 & $0.3962(14)$ & $0.0325(13)$ & $0.2015(9)$ & $0.032(3)^{*}$ \\
C6 & $0.53750(9)$ & $0.18006(9)$ & $0.28052(6)$ & $0.01830(19)$ \\
C7 & $0.49871(9)$ & $0.44227(9)$ & $0.30059(6)$ & $0.01918(19)$ \\
C8 & $0.62953(9)$ & $0.43608(9)$ & $0.37231(5)$ & $0.01762(19)$ \\
C9 & $0.31974(9)$ & $0.30696(10)$ & $0.19483(6)$ & $0.0233(2)$ \\
H9A & $0.2631(14)$ & $0.3931(15)$ & $0.2067(9)$ & $0.033(3)^{*}$ \\
H9B & $0.2651(13)$ & $0.2186(14)$ & $0.2059(8)$ & $0.029(3)^{*}$ \\
C10 & $0.35231(12)$ & $0.31217(12)$ & $0.09942(7)$ & $0.0316(2)$ \\
H10A & $0.4106(16)$ & $0.4010(16)$ & $0.0903(10)$ & $0.043(4)^{*}$ \\
H10B & $0.4032(16)$ & $0.2247(17)$ & $0.0845(10)$ & $0.047(4)^{*}$ \\
H10C & $0.2620(16)$ & $0.3135(15)$ & $0.0563(11)$ & $0.048(4)^{*}$ \\
C11 & $0.66935(9)$ & $0.56627(9)$ & $0.43138(6)$ & $0.01939(19)$ \\
C12 & $0.56472(10)$ & $0.66442(10)$ & $0.45417(6)$ & $0.0239(2)$ \\
H12 & $0.4642(14)$ & $0.6541(13)$ & $0.4277(9)$ & $0.029(3)^{*}$ \\
C13 & $0.60553(11)$ & $0.77375(11)$ & $0.51836(7)$ & $0.0288(2)$ \\
H13 & $0.5343(14)$ & $0.8431(14)$ & $0.5340(9)$ & $0.034(3)^{*}$ \\
C14 & $0.75007(12)$ & $0.78690(11)$ & $0.55996(7)$ & $0.0311(2)$ \\
H14 & $0.7760(14)$ & $0.8637(16)$ & $0.6076(10)$ & $0.040(3)^{*}$ \\
& & & &
\end{tabular}




\begin{tabular}{lllll}
$\mathrm{C} 15$ & $0.85551(12)$ & $0.69263(11)$ & $0.53570(7)$ & $0.0313(2)$ \\
$\mathrm{H} 15$ & $0.9599(15)$ & $0.7034(14)$ & $0.5636(10)$ & $0.041(4)^{*}$ \\
C16 & $0.81542(10)$ & $0.58303(10)$ & $0.47181(7)$ & $0.0258(2)$ \\
$\mathrm{H} 16$ & $0.8876(14)$ & $0.5150(15)$ & $0.4564(8)$ & $0.034(3)^{*}$ \\
\hline
\end{tabular}

Atomic displacement parameters $\left(\AA^{2}\right)$

\begin{tabular}{lllllll}
\hline & $U^{11}$ & $U^{22}$ & $U^{33}$ & $U^{12}$ & $U^{13}$ & $U^{23}$ \\
\hline $\mathrm{O} 1$ & $0.0285(3)$ & $0.0206(3)$ & $0.0317(4)$ & $0.0062(2)$ & $-0.0059(3)$ & $-0.0015(3)$ \\
$\mathrm{N} 1$ & $0.0178(3)$ & $0.0182(4)$ & $0.0182(3)$ & $-0.0006(2)$ & $0.0006(3)$ & $-0.0008(2)$ \\
$\mathrm{N} 2$ & $0.0208(3)$ & $0.0179(3)$ & $0.0192(4)$ & $0.0000(2)$ & $0.0017(3)$ & $0.0004(2)$ \\
$\mathrm{C} 1$ & $0.0208(4)$ & $0.0172(4)$ & $0.0180(4)$ & $0.0004(3)$ & $0.0042(3)$ & $0.0004(3)$ \\
$\mathrm{C} 2$ & $0.0256(4)$ & $0.0208(4)$ & $0.0223(4)$ & $0.0040(3)$ & $0.0043(3)$ & $0.0021(3)$ \\
$\mathrm{C} 3$ & $0.0346(5)$ & $0.0181(4)$ & $0.0254(5)$ & $0.0048(3)$ & $0.0103(4)$ & $0.0023(3)$ \\
C4 & $0.0361(5)$ & $0.0177(4)$ & $0.0264(5)$ & $-0.0035(3)$ & $0.0097(4)$ & $-0.0020(3)$ \\
C5 & $0.0279(4)$ & $0.0204(4)$ & $0.0226(4)$ & $-0.0041(3)$ & $0.0039(3)$ & $-0.0012(3)$ \\
C6 & $0.0215(4)$ & $0.0169(4)$ & $0.0172(4)$ & $-0.0006(3)$ & $0.0051(3)$ & $0.0008(3)$ \\
C7 & $0.0192(4)$ & $0.0178(4)$ & $0.0200(4)$ & $0.0001(3)$ & $0.0015(3)$ & $-0.0010(3)$ \\
C8 & $0.0182(4)$ & $0.0172(4)$ & $0.0170(4)$ & $-0.0010(3)$ & $0.0014(3)$ & $0.0000(3)$ \\
C9 & $0.0190(4)$ & $0.0247(4)$ & $0.0244(4)$ & $-0.0025(3)$ & $-0.0021(3)$ & $-0.0022(3)$ \\
C10 & $0.0377(5)$ & $0.0328(5)$ & $0.0216(5)$ & $-0.0009(4)$ & $-0.0036(4)$ & $-0.0013(4)$ \\
C11 & $0.0234(4)$ & $0.0164(4)$ & $0.0178(4)$ & $-0.0024(3)$ & $0.0018(3)$ & $0.0005(3)$ \\
C12 & $0.0258(4)$ & $0.0226(4)$ & $0.0242(4)$ & $-0.0026(3)$ & $0.0066(3)$ & $-0.0014(3)$ \\
C13 & $0.0387(5)$ & $0.0233(4)$ & $0.0271(5)$ & $-0.0036(4)$ & $0.0137(4)$ & $-0.0047(4)$ \\
C14 & $0.0465(6)$ & $0.0249(5)$ & $0.0217(5)$ & $-0.0114(4)$ & $0.0054(4)$ & $-0.0050(4)$ \\
C15 & $0.0337(5)$ & $0.0284(5)$ & $0.0284(5)$ & $-0.0075(4)$ & $-0.0052(4)$ & $-0.0023(4)$ \\
C16 & $0.0251(4)$ & $0.0227(4)$ & $0.0272(5)$ & $-0.0014(3)$ & $-0.0023(3)$ & $-0.0015(3)$ \\
& & & & & & \\
\hline
\end{tabular}

Geometric parameters $\left(A,{ }^{\circ}\right)$

\begin{tabular}{llll}
\hline $\mathrm{O} 1-\mathrm{C} 7$ & $1.2299(10)$ & $\mathrm{C} 9-\mathrm{C} 10$ & $1.5156(14)$ \\
$\mathrm{N} 1-\mathrm{C} 7$ & $1.3791(10)$ & $\mathrm{C} 9-\mathrm{H} 9 \mathrm{~A}$ & $0.972(13)$ \\
$\mathrm{N} 1-\mathrm{C} 6$ & $1.3936(10)$ & $\mathrm{C} 9-\mathrm{H} 9 \mathrm{~B}$ & $0.975(13)$ \\
$\mathrm{N} 1-\mathrm{C} 9$ & $1.4732(11)$ & $\mathrm{C} 10-\mathrm{H} 10 \mathrm{~A}$ & $0.990(14)$ \\
$\mathrm{N} 2-\mathrm{C} 8$ & $1.2983(11)$ & $\mathrm{C} 10-\mathrm{H} 10 \mathrm{~B}$ & $0.966(15)$ \\
$\mathrm{N} 2-\mathrm{C} 1$ & $1.3846(11)$ & $\mathrm{C} 10-\mathrm{H} 10 \mathrm{C}$ & $0.972(16)$ \\
$\mathrm{C} 1-\mathrm{C} 2$ & $1.4063(11)$ & $\mathrm{C} 11-\mathrm{C} 12$ & $1.3976(12)$ \\
$\mathrm{C} 1-\mathrm{C} 6$ & $1.4071(12)$ & $\mathrm{C} 11-\mathrm{C} 16$ & $1.3983(12)$ \\
$\mathrm{C} 2-\mathrm{C} 3$ & $1.3770(13)$ & $\mathrm{C} 12-\mathrm{C} 13$ & $1.3920(13)$ \\
$\mathrm{C} 2-\mathrm{H} 2$ & $0.980(13)$ & $\mathrm{C} 12-\mathrm{H} 12$ & $0.955(12)$ \\
$\mathrm{C} 3-\mathrm{C} 4$ & $1.3995(14)$ & $\mathrm{C} 13-\mathrm{C} 14$ & $1.3875(15)$ \\
$\mathrm{C} 3-\mathrm{H} 3$ & $0.995(12)$ & $\mathrm{C} 13-\mathrm{H} 13$ & $0.967(12)$ \\
$\mathrm{C} 4-\mathrm{C} 5$ & $1.3834(13)$ & $\mathrm{C} 14-\mathrm{C} 15$ & $1.3893(16)$ \\
$\mathrm{C} 4-\mathrm{H} 4$ & $0.985(14)$ & $\mathrm{C} 14-\mathrm{H} 14$ & $1.000(15)$ \\
$\mathrm{C} 5-\mathrm{C} 6$ & $1.4065(11)$ & $\mathrm{C} 15-\mathrm{C} 16$ & $0.994(14)$ \\
$\mathrm{C} 5-\mathrm{H} 5$ & $1.006(12)$ & $\mathrm{C} 15-\mathrm{H} 15$ & $0.964(13)$ \\
$\mathrm{C} 7-\mathrm{C} 8$ & $1.4872(11)$ & $\mathrm{C} 16-\mathrm{H} 16$ & \\
$\mathrm{C} 8-\mathrm{C} 11$ & $1.4864(11)$ & &
\end{tabular}




\begin{tabular}{|c|c|c|c|}
\hline $\mathrm{C} 7-\mathrm{N} 1-\mathrm{C} 6$ & $122.16(7)$ & $\mathrm{N} 1-\mathrm{C} 9-\mathrm{H} 9 \mathrm{~A}$ & $107.0(8)$ \\
\hline $\mathrm{C} 7-\mathrm{N} 1-\mathrm{C} 9$ & $117.14(7)$ & $\mathrm{C} 10-\mathrm{C} 9-\mathrm{H} 9 \mathrm{~A}$ & $110.2(8)$ \\
\hline $\mathrm{C} 6-\mathrm{N} 1-\mathrm{C} 9$ & $120.63(7)$ & $\mathrm{N} 1-\mathrm{C} 9-\mathrm{H} 9 \mathrm{~B}$ & $107.3(7)$ \\
\hline $\mathrm{C} 8-\mathrm{N} 2-\mathrm{C} 1$ & $119.29(7)$ & $\mathrm{C} 10-\mathrm{C} 9-\mathrm{H} 9 \mathrm{~B}$ & $112.1(7)$ \\
\hline $\mathrm{N} 2-\mathrm{C} 1-\mathrm{C} 2$ & $118.35(8)$ & $\mathrm{H} 9 \mathrm{~A}-\mathrm{C} 9-\mathrm{H} 9 \mathrm{~B}$ & $108.4(11)$ \\
\hline $\mathrm{N} 2-\mathrm{C} 1-\mathrm{C} 6$ & $121.76(7)$ & $\mathrm{C} 9-\mathrm{C} 10-\mathrm{H} 10 \mathrm{~A}$ & $110.7(9)$ \\
\hline $\mathrm{C} 2-\mathrm{C} 1-\mathrm{C} 6$ & $119.72(8)$ & $\mathrm{C} 9-\mathrm{C} 10-\mathrm{H} 10 \mathrm{~B}$ & $112.0(9)$ \\
\hline $\mathrm{C} 3-\mathrm{C} 2-\mathrm{C} 1$ & $120.54(9)$ & $\mathrm{H} 10 \mathrm{~A}-\mathrm{C} 10-\mathrm{H} 10 \mathrm{~B}$ & 109.7 (12) \\
\hline $\mathrm{C} 3-\mathrm{C} 2-\mathrm{H} 2$ & $122.3(7)$ & $\mathrm{C} 9-\mathrm{C} 10-\mathrm{H} 10 \mathrm{C}$ & $110.6(9)$ \\
\hline $\mathrm{C} 1-\mathrm{C} 2-\mathrm{H} 2$ & $117.2(7)$ & $\mathrm{H} 10 \mathrm{~A}-\mathrm{C} 10-\mathrm{H} 10 \mathrm{C}$ & $109.0(12)$ \\
\hline $\mathrm{C} 2-\mathrm{C} 3-\mathrm{C} 4$ & $119.51(8)$ & $\mathrm{H} 10 \mathrm{~B}-\mathrm{C} 10-\mathrm{H} 10 \mathrm{C}$ & $104.7(12)$ \\
\hline $\mathrm{C} 2-\mathrm{C} 3-\mathrm{H} 3$ & $121.2(7)$ & $\mathrm{C} 12-\mathrm{C} 11-\mathrm{C} 16$ & $118.94(8)$ \\
\hline $\mathrm{C} 4-\mathrm{C} 3-\mathrm{H} 3$ & $119.3(7)$ & $\mathrm{C} 12-\mathrm{C} 11-\mathrm{C} 8$ & $122.45(8)$ \\
\hline $\mathrm{C} 5-\mathrm{C} 4-\mathrm{C} 3$ & $121.16(8)$ & $\mathrm{C} 16-\mathrm{C} 11-\mathrm{C} 8$ & $118.36(8)$ \\
\hline $\mathrm{C} 5-\mathrm{C} 4-\mathrm{H} 4$ & $119.7(8)$ & $\mathrm{C} 13-\mathrm{C} 12-\mathrm{C} 11$ & $120.11(9)$ \\
\hline $\mathrm{C} 3-\mathrm{C} 4-\mathrm{H} 4$ & $119.2(8)$ & $\mathrm{C} 13-\mathrm{C} 12-\mathrm{H} 12$ & $119.7(7)$ \\
\hline $\mathrm{C} 4-\mathrm{C} 5-\mathrm{C} 6$ & $119.67(9)$ & $\mathrm{C} 11-\mathrm{C} 12-\mathrm{H} 12$ & $120.1(7)$ \\
\hline $\mathrm{C} 4-\mathrm{C} 5-\mathrm{H} 5$ & $120.2(7)$ & $\mathrm{C} 14-\mathrm{C} 13-\mathrm{C} 12$ & $120.51(9)$ \\
\hline $\mathrm{C} 6-\mathrm{C} 5-\mathrm{H} 5$ & $120.0(7)$ & $\mathrm{C} 14-\mathrm{C} 13-\mathrm{H} 13$ & $118.7(8)$ \\
\hline $\mathrm{N} 1-\mathrm{C} 6-\mathrm{C} 5$ & $122.78(8)$ & $\mathrm{C} 12-\mathrm{C} 13-\mathrm{H} 13$ & $120.8(8)$ \\
\hline $\mathrm{N} 1-\mathrm{C} 6-\mathrm{C} 1$ & $117.87(7)$ & $\mathrm{C} 13-\mathrm{C} 14-\mathrm{C} 15$ & $119.73(9)$ \\
\hline $\mathrm{C} 5-\mathrm{C} 6-\mathrm{C} 1$ & $119.34(7)$ & $\mathrm{C} 13-\mathrm{C} 14-\mathrm{H} 14$ & $119.0(8)$ \\
\hline $\mathrm{O} 1-\mathrm{C} 7-\mathrm{N} 1$ & $121.54(8)$ & $\mathrm{C} 15-\mathrm{C} 14-\mathrm{H} 14$ & $121.2(8)$ \\
\hline $\mathrm{O} 1-\mathrm{C} 7-\mathrm{C} 8$ & $123.36(7)$ & $\mathrm{C} 14-\mathrm{C} 15-\mathrm{C} 16$ & $120.02(9)$ \\
\hline $\mathrm{N} 1-\mathrm{C} 7-\mathrm{C} 8$ & $115.10(7)$ & $\mathrm{C} 14-\mathrm{C} 15-\mathrm{H} 15$ & $120.3(8)$ \\
\hline $\mathrm{N} 2-\mathrm{C} 8-\mathrm{C} 11$ & $117.24(7)$ & $\mathrm{C} 16-\mathrm{C} 15-\mathrm{H} 15$ & $119.7(8)$ \\
\hline $\mathrm{N} 2-\mathrm{C} 8-\mathrm{C} 7$ & $122.87(7)$ & $\mathrm{C} 15-\mathrm{C} 16-\mathrm{C} 11$ & $120.64(9)$ \\
\hline $\mathrm{C} 11-\mathrm{C} 8-\mathrm{C} 7$ & $119.89(7)$ & $\mathrm{C} 15-\mathrm{C} 16-\mathrm{H} 16$ & $120.3(8)$ \\
\hline $\mathrm{N} 1-\mathrm{C} 9-\mathrm{C} 10$ & $111.67(7)$ & $\mathrm{C} 11-\mathrm{C} 16-\mathrm{H} 16$ & $119.0(8)$ \\
\hline $\mathrm{C} 8-\mathrm{N} 2-\mathrm{C} 1-\mathrm{C} 2$ & $-177.53(8)$ & $\mathrm{C} 1-\mathrm{N} 2-\mathrm{C} 8-\mathrm{C} 11$ & $172.67(7)$ \\
\hline $\mathrm{C} 8-\mathrm{N} 2-\mathrm{C} 1-\mathrm{C} 6$ & $-2.33(12)$ & $\mathrm{C} 1-\mathrm{N} 2-\mathrm{C} 8-\mathrm{C} 7$ & $-6.49(12)$ \\
\hline $\mathrm{N} 2-\mathrm{C} 1-\mathrm{C} 2-\mathrm{C} 3$ & $173.30(8)$ & $\mathrm{O} 1-\mathrm{C} 7-\mathrm{C} 8-\mathrm{N} 2$ & $-168.24(9)$ \\
\hline $\mathrm{C} 6-\mathrm{C} 1-\mathrm{C} 2-\mathrm{C} 3$ & $-2.01(13)$ & $\mathrm{N} 1-\mathrm{C} 7-\mathrm{C} 8-\mathrm{N} 2$ & $11.27(12)$ \\
\hline $\mathrm{C} 1-\mathrm{C} 2-\mathrm{C} 3-\mathrm{C} 4$ & $0.51(14)$ & $\mathrm{O} 1-\mathrm{C} 7-\mathrm{C} 8-\mathrm{C} 11$ & $12.63(13)$ \\
\hline $\mathrm{C} 2-\mathrm{C} 3-\mathrm{C} 4-\mathrm{C} 5$ & $1.36(14)$ & $\mathrm{N} 1-\mathrm{C} 7-\mathrm{C} 8-\mathrm{C} 11$ & $-167.87(7)$ \\
\hline $\mathrm{C} 3-\mathrm{C} 4-\mathrm{C} 5-\mathrm{C} 6$ & $-1.71(14)$ & $\mathrm{C} 7-\mathrm{N} 1-\mathrm{C} 9-\mathrm{C} 10$ & $98.17(9)$ \\
\hline $\mathrm{C} 7-\mathrm{N} 1-\mathrm{C} 6-\mathrm{C} 5$ & $178.92(8)$ & $\mathrm{C} 6-\mathrm{N} 1-\mathrm{C} 9-\mathrm{C} 10$ & $-78.78(10)$ \\
\hline $\mathrm{C} 9-\mathrm{N} 1-\mathrm{C} 6-\mathrm{C} 5$ & $-4.29(13)$ & $\mathrm{N} 2-\mathrm{C} 8-\mathrm{C} 11-\mathrm{C} 12$ & $-148.33(9)$ \\
\hline $\mathrm{C} 7-\mathrm{N} 1-\mathrm{C} 6-\mathrm{C} 1$ & $-0.46(12)$ & $\mathrm{C} 7-\mathrm{C} 8-\mathrm{C} 11-\mathrm{C} 12$ & $30.85(12)$ \\
\hline $\mathrm{C} 9-\mathrm{N} 1-\mathrm{C} 6-\mathrm{C} 1$ & $176.34(7)$ & $\mathrm{N} 2-\mathrm{C} 8-\mathrm{C} 11-\mathrm{C} 16$ & $25.81(12)$ \\
\hline $\mathrm{C} 4-\mathrm{C} 5-\mathrm{C} 6-\mathrm{N} 1$ & $-179.18(8)$ & $\mathrm{C} 7-\mathrm{C} 8-\mathrm{C} 11-\mathrm{C} 16$ & $-155.00(8)$ \\
\hline $\mathrm{C} 4-\mathrm{C} 5-\mathrm{C} 6-\mathrm{C} 1$ & $0.19(13)$ & $\mathrm{C} 16-\mathrm{C} 11-\mathrm{C} 12-\mathrm{C} 13$ & $-2.08(14)$ \\
\hline $\mathrm{N} 2-\mathrm{C} 1-\mathrm{C} 6-\mathrm{N} 1$ & $5.90(12)$ & $\mathrm{C} 8-\mathrm{C} 11-\mathrm{C} 12-\mathrm{C} 13$ & $172.04(8)$ \\
\hline $\mathrm{C} 2-\mathrm{C} 1-\mathrm{C} 6-\mathrm{N} 1$ & $-178.96(8)$ & $\mathrm{C} 11-\mathrm{C} 12-\mathrm{C} 13-\mathrm{C} 14$ & $0.30(14)$ \\
\hline $\mathrm{N} 2-\mathrm{C} 1-\mathrm{C} 6-\mathrm{C} 5$ & $-173.50(8)$ & $\mathrm{C} 12-\mathrm{C} 13-\mathrm{C} 14-\mathrm{C} 15$ & $1.64(15)$ \\
\hline $\mathrm{C} 2-\mathrm{C} 1-\mathrm{C} 6-\mathrm{C} 5$ & $1.64(12)$ & $\mathrm{C} 13-\mathrm{C} 14-\mathrm{C} 15-\mathrm{C} 16$ & $-1.78(15)$ \\
\hline
\end{tabular}


supporting information

\begin{tabular}{llll}
$\mathrm{C} 6-\mathrm{N} 1-\mathrm{C} 7-\mathrm{O} 1$ & $172.14(8)$ & $\mathrm{C} 14-\mathrm{C} 15-\mathrm{C} 16-\mathrm{C} 11$ & $-0.03(15)$ \\
$\mathrm{C} 9-\mathrm{N} 1-\mathrm{C} 7-\mathrm{O} 1$ & $-4.76(13)$ & $\mathrm{C} 12-\mathrm{C} 11-\mathrm{C} 16-\mathrm{C} 15$ & $1.95(14)$ \\
$\mathrm{C} 6-\mathrm{N} 1-\mathrm{C} 7-\mathrm{C} 8$ & $-7.37(12)$ & $\mathrm{C} 8-\mathrm{C} 11-\mathrm{C} 16-\mathrm{C} 15$ & $-172.41(9)$ \\
$\mathrm{C} 9-\mathrm{N} 1-\mathrm{C} 7-\mathrm{C} 8$ & $175.73(7)$ & & \\
\hline
\end{tabular}

Hydrogen-bond geometry $\left(A,{ }^{\circ}\right)$

\begin{tabular}{lllll}
\hline$D-\mathrm{H} \cdots A$ & $D-\mathrm{H}$ & $\mathrm{H} \cdots A$ & $D \cdots A$ & $D-\mathrm{H} \cdots A$ \\
\hline $\mathrm{C} 9-\mathrm{H} 9 B \cdots \mathrm{O} 1^{\mathrm{i}}$ & $0.975(13)$ & $2.396(13)$ & $3.3340(11)$ & $161.3(10)$ \\
\hline
\end{tabular}

Symmetry code: (i) $-x+1 / 2, y-1 / 2,-z+1 / 2$. 